\title{
Piezoelectricity from Elastic and Dielectric Measurements on Unpoled Ferroelectrics
}

\author{
Francesco Cordero $^{a} * \mathbb{D}$ \\ ${ }^{a}$ Istituto di Struttura della Materia (ISM-CNR), Area della Ricerca di Roma-Tor Vergata, Via del Fosso \\ del Cavaliere 100, I-00133 Roma, Italy
}

Received: September 24, 2017; Revised: February 15, 2018; Accepted: April 20, 2018

\begin{abstract}
A method is described to assess the piezoelectric response of ferroelectric materials from the temperature dependence of the elastic moduli and dielectric constant of unpoled samples. The limits and advantages over the traditional methods on poled samples are discussed. A quantitative check is made by comparing the piezoelectric softening measured below the ferroelectric transition in the Young's modulus of unpoled ceramic $\mathrm{BaTiO}_{3}$, with the appropriate angular average of the single crystal material constants from the literature. The softening effect of porosity has been taken into account by comparing the moduli of samples with different densities.
\end{abstract}

Keywords: ferroelectricity, piezoelectricity, elastic constants.

\section{Introduction}

The ferroelectric (FE) materials are mostly used in applications exploiting the piezoelectric effect, a change of their polarization $\mathbf{P}$ and hence the generation of an electric field, under application of a stress $\sigma$ (direct effect), and the generation of strain $\varepsilon$ under application of an electric field $\mathbf{E}$ (converse effect) ${ }^{1}$. The magnitude of the effect is quantified by the piezoelectric tensor $d_{i j k}=d P_{i} / d \sigma_{j k}$ and $d^{+}{ }_{i j k}$ $=d \varepsilon_{i j} / d E_{k}$, where the cross indicates the transposed ${ }^{2}$. The measurement of the piezoelectric constants on ceramics may vary considerably depending on the sample microstructure, content of defects, and experimental conditions. Apart from the indetermination related to the field dependent extrinsic contribution from the domain wall motion, a major source of error with respect to the intrinsic value is incomplete poling $^{3}$. In fact, the magnitude of the piezoelectric effect is proportional to the polarization, and a ceramic has to be poled in an intense electric field, in order to orient as parallel as possible to the field the spontaneous polarization, otherwise randomly oriented into domains. Such a process is often incomplete, due to the elastic clamping of non- $180^{\circ}$ switching, or due to defects creating local electric fields or pinning the domain walls or producing electric conductivity that shorts the electrodes.

The elastic and anelastic measurements are generally of side interest in the research on FE materials, but it has recently been shown that it is possible to derive information on the piezoelectric coupling in ceramic samples, without the need of poling them, from the softening, that in the FE phase exceeds the compliance of the paraelectric (PE) phase ${ }^{4}$. In unpoled ceramic samples, where the depolarization field should be negligible or at least minimal ${ }^{1}$, the elastic softening would be a good indicator of the maximum potential piezoelectric coupling in the fully poled material. Here the formula expressing the softening in terms of the piezoelectric and dielectric constants ${ }^{4}$ is derived using the formalism of anelastic relaxation, and is validated by comparing the softening of the Young's modulus of unpoled ceramic $\mathrm{BaTiO}_{3}$ with the appropriate angular average of the piezoelectric and dielectric tensors from the literature.

\section{Experimental}

The ceramic $\mathrm{BaTiO}_{3}$ samples were prepared by conventional solid state reaction in different laboratories, as described in Ref. 5. The high Curie temperatures (400 $\mathrm{K})$ indicate the good quality of the samples. Sample BT1 had length and thickness $33 \times 1.1 \mathrm{~mm}$ and a relative density of $90 \%$, measured with Archimede's method; sample BT2 had $46 \times 0.5 \mathrm{~mm}$ and relative density of $88 \%$. The dynamic Young's moduli were measured by electrostatically exciting the free flexural resonance of the bars (1-6 kHz), suspended on thin thermocouple wires in vacuum. 


\section{Results and Discussion}

\subsection{Piezoelectric softening}

In general, the softening $\Delta s$ of the compliance $s$ due to anelastic relaxation can be written as

$$
\Delta s=\frac{d \varepsilon^{a n}}{d \sigma}=\frac{d \varepsilon^{a n}}{d \xi} \frac{d \xi}{d \sigma}
$$

where $\varepsilon$ and $\sigma$ are strain and stress, and $\xi$ is an internal variable, such as a combination of defect populations, which is coupled to stress and anelastic strain, e.g. through the elastic dipole tensor. We show now that a similar expression can be derived for a FE, with $\xi \equiv P$. This can be done by writing the elastic Gibbs energy, $G=F-\sigma \varepsilon$, as ${ }^{6}$

$$
G=F(P)-\frac{1}{2} s^{0} \sigma^{2}-Q \sigma P^{2}
$$

where $F(P)$ is the free energy of the unstressed material (not necessarily the Landau expansion in powers of $P$ ), the second term is the elastic energy in the PE state, with $P=0$, and $-Q \sigma P^{2}$ is the first permissible term containing powers of both $\sigma$ and $P$, called electrostrictive. In fact, a piezoelectric term - $g \sigma P$ would not satisfy the inversion symmetry in the PE phase, but we will now see that piezoelectricity in the FE phase is a consequence of electrostriction. The strain is deduced by reminding that $d G=-S d T-\varepsilon d \sigma+E d P$, so that

$$
\varepsilon=-\frac{\partial G}{\partial \sigma}=s^{0} \sigma+Q P^{2} \rightarrow \frac{\partial \varepsilon}{\partial P}=2 Q P
$$

where the first term is elastic and the second electrostrictive. To complete our task, we need to evaluate $d P / d \sigma=d$. The equilibrium polarization under the action of stress minimizes $G$, and therefore is solution of $0=\partial F / \partial P-2 Q \sigma P$. By differentiating with respect to $\sigma$ and solving with respect to $d P / d \sigma$ for $\sigma \rightarrow 0$ we find

$$
d=\frac{d P}{d \sigma}=2 \epsilon Q P
$$

where we used the fact that $\partial^{2} F / \partial^{2} P=\chi^{-1}$ and for a FE $\chi \simeq$ $\epsilon$, the dielectric permittivity. By putting together the above results we find

$$
\Delta s^{\text {piezo }}=\frac{d \varepsilon}{d \sigma}=\frac{d \varepsilon}{d P} \frac{d P}{d \sigma}=4 \epsilon Q^{2} P^{2}=\frac{d^{2}}{\epsilon},
$$

a softening of totally electrostrictive origin, which occurs only in the FE phase with $P>0$, where electrostriction becomes piezoelectricity with $d \propto P$. The above steps are illustrated in Fig. 1.

This result is already contained in the paper of Devonshire, deducing the properties of $\mathrm{BaTiO}_{3}$ from an expansion of the free energy in powers of $P$ up to the sixth order ${ }^{7}$, and in the subsequent literature of the same kind. The physical meaning and implications of those results, however, are

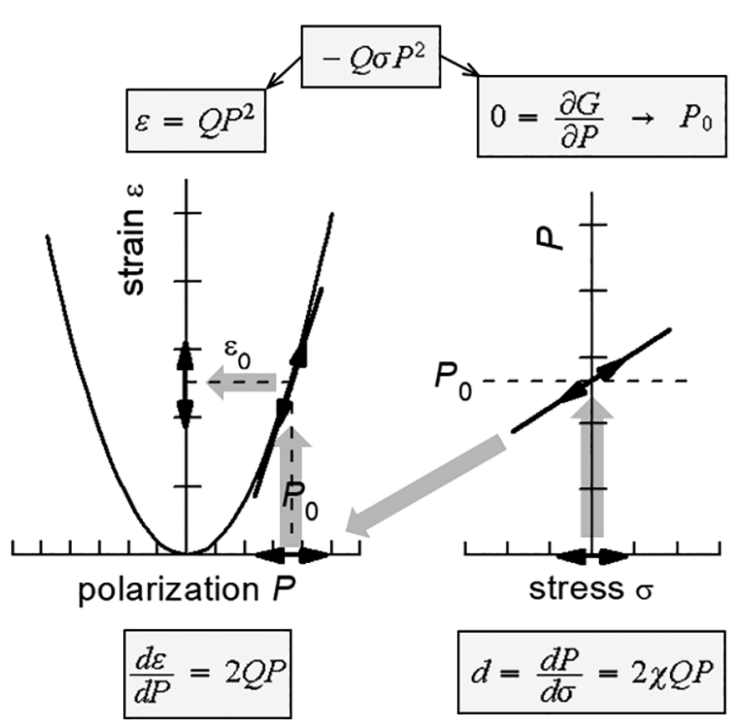

Figure 1. Scheme of how the electrostrictive coupling produces the piezoelectric softening in the FE phase.

hidden in the complicated expressions of the elastic responses, generally presented as curves calculated for specific sets of the parameters.

\subsection{Validation of the elastic method for determining the piezoelectric coupling on unpoled ceramic samples}

A method for evaluating the piezoelectric response from elastic and dielectric measurements of an unpoled ceramic may seem quite unlikely, but Eq. (4) clearly supports it. On the other hand, the formula has been derived in the framework of the thermodynamic theory of phase transitions, which for example ignores the fluctuations of the polarization, and therefore needs testing its validity. The conceptually most straightforward test would be with Resonant Ultrasound Spectroscopy (RUS) and dielectric measurements on a same single crystal, from which the complete $s, d$, and $\epsilon$ tensors can be measured. In the present case neither single crystals nor the RUS techniques are available, and the more commonly available measurement of the Young's modulus $E=s^{-1}$ of ceramic samples is considered. In order to extract the set of $s, d$, and $\epsilon$ constants of a ceramic material, it is necessary to use several samples with different geometries (only the $s$ tensor can be fully determined with a single RUS measurement ${ }^{3}$ ), which introduces great uncertainties ${ }^{3}$. Therefore, the material of choice is $\mathrm{BaTiO}_{3}$, a well characterized material for which single crystal data are available in the literature, and the quality of the material can promptly be assessed from the Curie temperature $T_{\mathrm{C}}$ and the shape of the elastic curves.

In order to compare the softening of the Young's modulus (uniaxial stress) of a ceramic with Eq. (4), it is necessary to perform an orientational average of that equation. This 
is done by applying to Eq. (4), written in tensorial form for the tetragonal phase of interest, the projector to the uniaxial stress in the generic direction $\hat{\boldsymbol{n}}^{2}$,

$$
\Delta s^{\text {piezo }}(\hat{\boldsymbol{n}} \hat{\boldsymbol{n}})=\hat{\boldsymbol{n}} \hat{\boldsymbol{n}}: \boldsymbol{d}^{+} \cdot \boldsymbol{\epsilon}^{-1} \cdot \boldsymbol{d}: \hat{\boldsymbol{n}} \hat{\boldsymbol{n}}
$$

For an untextured unpoled ceramic, the directions of the local spontaneous polarizations are truly random, unlike the case of poled samples, so that the orientational average is simply $\left\langle n_{z}^{2}\right\rangle=\frac{1}{\pi} \int^{\pi} d \theta \cos ^{2} \theta=\frac{1}{3},\left\langle n_{z}^{4}\right\rangle=\frac{1}{5}$, and $\left\langle n^{2}{ }_{x}\right\rangle=\left\langle n^{2}{ }_{y}\right\rangle$, $n_{x}^{2}+n_{y}^{2}+n_{z}^{2} \stackrel{=}{=} 1$ can be used. Using the Voigt matrix notation for the tensors, the average in the $\mathrm{T}$ phase results

$\Delta s^{\text {piezo,T }}=\frac{8 d_{31}^{2}+4 d_{31} d_{33}+3 d_{33}^{2}}{15 \epsilon_{33}}+\frac{2 d_{15}^{2}}{15 \epsilon_{11}}$

Yet, in order to compare the results from porous ceramic samples with those of $100 \%$ dense single crystals, it is necessary to assess the effect of porosity on the measured compliance.

Figure 2 shows the Young's moduli of the two samples BT1 and BT2, with porosities of $10 \%$ and $12 \%$ respectively, together with the data of the denser sample ( $4 \%$ porosity) from Ref. 8, here labelled BT0. We assume that BT0 is close enough to a fully dense material, and take as reference its extrapolated maximum modulus $E_{\text {max }}=200 \mathrm{GPa}$ in the PE phase. Clearly, the compliance is not linearly softened by porosity, and the geometry of the pores plays a primary role. Luckily, after rescaling the curves of BT1 and BT2 onto BT0 in the PE phase, all the curves agree reasonably well also in the FE phase (Fig. 2b). This allows us to use the curve of our denser sample BT1, after rescaling it by a factor 200/158.

Barium titanate has the complication that the tetragonal FE transition is followed by other transitions, where the direction of the polarization changes and the symmetry becomes orthorhombic and then rhombohedral (Figs. 2,3). In addition, the fluctuations of the polarization are particularly important in $\mathrm{BaTiO}_{3}$, and produce a precursor softening that extends for $200 \mathrm{~K}$ above $T_{\mathrm{C}}$. The piezoelectric coefficients well above $T_{\mathrm{C}}$ are null, and, according to Eq. (4), also $\Delta s^{\text {piezo }}$ should be null. At present, it is left as an open issue whether the precursor softening above $T_{\mathrm{C}}$ should be considered as piezoelectric softening or not. In either case, however, it is clear that also above the $\mathrm{O} / \mathrm{T}$ transition a precursor softening is expected, this time from the transverse fluctuations of $\mathbf{P}$, which is going to change direction.

It is therefore safe to test Eq. (6), not taking into account fluctuations, far from the transition temperatures; the best choice is the plateau of $s(T)$ around $373 \mathrm{~K}$.

Finally, the contribution $s^{0}$ at constant $\mathbf{P}$ must be evaluated, in order to be subtracted. Though it cannot be measured
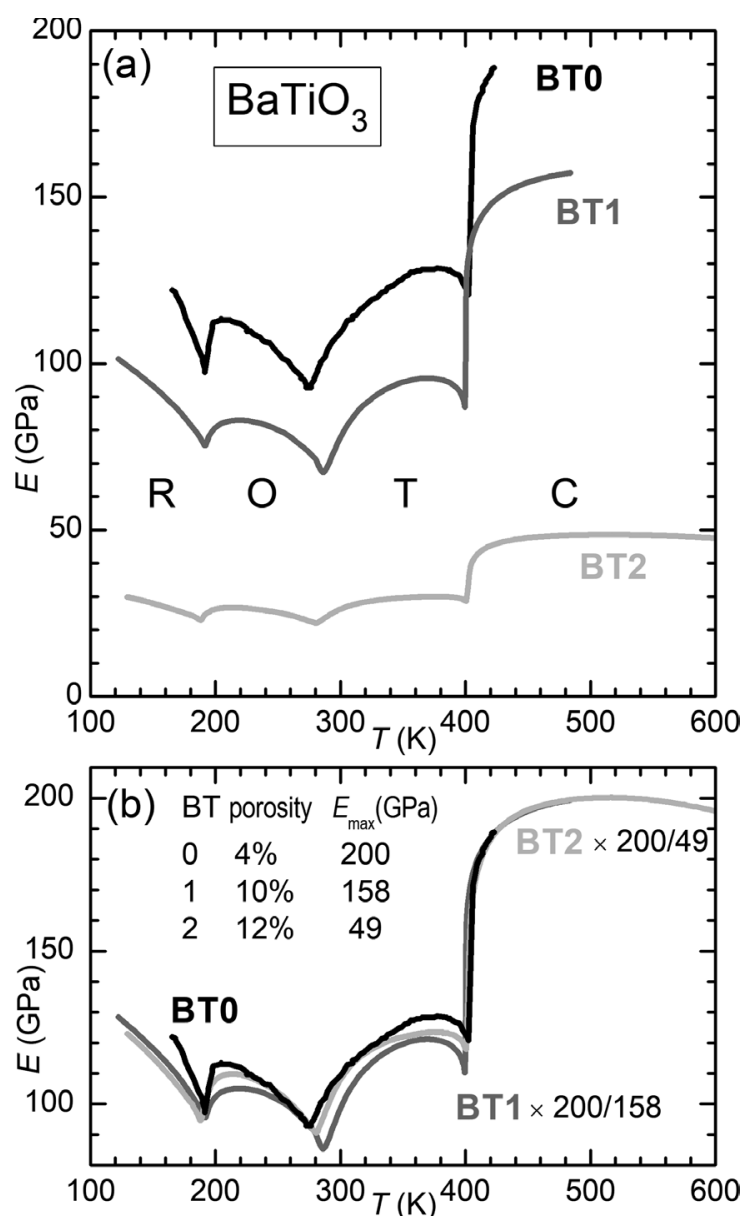

Figure 2. Young's moduli of samples BT0,1,2 of ceramic $\mathrm{BaTiO}_{3}$; the structural phases are indicated as $\mathrm{C}=$ cubic, $\mathrm{T}=$ tetragonal, $\mathrm{O}=$ orthorhombic, $\mathrm{R}=$ rhombohedral. In (b) the BT 1 and BT2 curves are multiplied by the factors indicated in the labels, in order to overlap with BT0 in the PE phase.

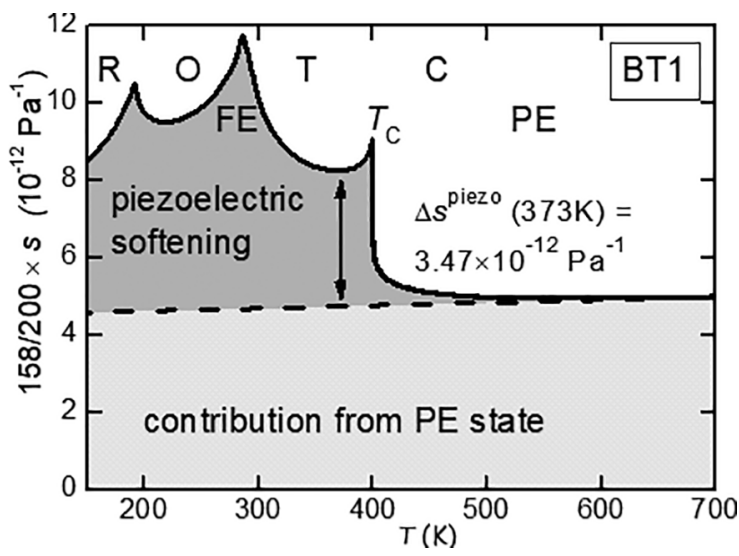

Figure 3. Reciprocal Young's modulus of BT1, normalized to $E_{\max }=200 \mathrm{GPa}$, with subtraction of the PE background compliance. 
directly, $s^{0}(T)$ must be a practically linear function of $T$ at least down to $100-200 \mathrm{~K}$, since, analogously to the thermal expansion, is an anharmonic lattice effect. This can be directly checked on $\mathrm{SrTiO}_{3}$, which remains cubic down to $105 \mathrm{~K}$. For $\mathrm{BaTiO}_{3}$, the slope of $s^{0}(T)$ must be extrapolated from above the range of the precursor fluctuations, namely above $700 \mathrm{~K}$, and the result is shown as a dashed line in Fig. 3.

The amplitude of the piezoelectric softening of BT1 at the plateau is then

$$
\Delta s^{\text {piezo }}(373 \mathrm{~K} ; \mathrm{BT} 1)=3.47 \times 10^{-12} \mathrm{~Pa}^{-1} .
$$

This value must be compared with Eq. (6), where the single crystal data at the same temperature are inserted. To our knowledge, the most reliable and recent set of materials constants of $\mathrm{BaTiO}_{3}$ is that calculated from a Landau free energy, that best reproduces the phase diagram and physical properties of $\mathrm{BaTiO}_{3}{ }^{9,10}$. Inserting those values in Eq. (6), we obtain

$$
\Delta s^{\text {piezo }}(373 \mathrm{~K} ; \mathrm{SC})=3.68 \times 10^{-12} \mathrm{~Pa}^{-1},
$$

Which differs from (7) of only $6 \%$. If curve BT0 is chosen, instead of BT1, with the smallest $\Delta s^{\text {piezo }}=3.0 \times 10^{-12} \mathrm{~Pa}^{-1}$, the deviation from the expected value (8) is still within $17 \%$.

This test of Eq. (6) is very satisfactory far from the FE transitions, but larger deviations are found on approaching both $T_{\mathrm{C}}$ and the transition to the $\mathrm{O}$ phase, advising caution for the application of this method as a quantitative tool for determining the piezoelectric coefficients too close to the transitions. The method is instead expected to be very useful in situations like the development of new or modified FE materials, which are still not optimized and therefore highly defective and hardly polarizable. In these cases, the piezoelectric softening is not affected by the sample conductivity or excessive coercive field, since poling is not necessary, and the magnitude of the softening below $T_{\mathrm{C}}$ is a good indicator whether the intrinsic piezoelectric coupling is improving or not. In such situations there is no need to compare $\Delta s^{\text {piezo }}$ with unknown intrinsic material constants through Eq. (6), and it is sufficient to compare the amplitudes of the softenings through $T_{C}$ of the samples with varying compositions or preparation procedures.

The use of Eq. (5) is particularly promising in conjunction with RUS experiments. Such measurements have already been used to extract the complete set of elastic and piezoelectric coefficients within the FE phase on poled ceramic samples ${ }^{3}$. The same measurements, repeated on unpoled samples and extended into the PE phase, would provide the complete set of elastic constants in both the FE and PE phase and the piezoelectric coefficients, without the uncertainty introduced by possibly incomplete poling.
A situation in which the softening below $T_{\mathrm{C}}$ is not indicative of the piezoelectric coupling is when the FE transition is accompanied by another structural instability, like octahedral tilting. A notable such example is $\mathrm{Na}_{1 / 2} \mathrm{Bi}_{1 / 2} \mathrm{TiO}_{3}$, whereas tilting in rhombohedral PZT does not affect the evaluation of the piezoelectric softening, because it occurs at much lower temperature than $T_{\mathrm{C}}$.

\section{Conclusions}

It is demonstrated that, within the usual thermodynamic treatment of ferroelectricity, the softening below the FE transition is totally of piezoelectric origin, and therefore, combined with the dielectric permittivity, can be used to evaluate the piezoelectric coupling. It does not require that the sample is poled, providing a method for evaluating the potential piezoelectric response of a material, using unpoled ceramic samples.

The method is tested by comparing the softening measured on ceramic samples of $\mathrm{BaTiO}_{3}$ with that expected using the known material constant.

\section{References}

1. Heywang W, Lubitz K, Wersing W, eds. Piezoelectricity Evolution and Future of a Technology. Berlin, Heidelberg: Springer-Verlag; 2008.

2. Sirotin YI, Shaskolskaya MP. Fundamentals of Crystal Physics. Moscow: Mir Publishers; 1982.

3. Tang L, Cao W. Temperature dependence of self-consistent full matrix material constants of lead zirconate titanate ceramics. Applied Physics Letters. 2015;106(5):052902.

4. Cordero F, Craciun F, Trequattrini F, Galassi C. Piezoelectric softening in ferroelectrics: Ferroelectric versus antiferroelectric $\mathrm{PbZr}_{1-\mathrm{x}} \mathrm{Ti}_{\mathrm{x}} \mathrm{O}_{3}$. Physical Review B. 2016;93(17):174111.

5. Cordero F, Langhammer HT, Müller T, Buscaglia V, Nanni P. Rotational instability of the electric polarization and divergence of the shear elastic compliance. Physical Review B. 2016;93(6):064111.

6. Strukov BA, Levanyuk AP. Ferroelectric Phenomena in Crystals. Berlin, Heidelberg: Springer-Verlag; 1998.

7. Devonshire AF. CIX. Theory of barium titanate-Part II. Philosophical Magazine. 1951;42:1065-1079.

8. Cheng BL, Gabbay M, Duffy W Jr, Fantozzi G. Mechanical loss and Young's modulus associated with phase transitions in barium titanate based ceramics. Journal of Materials Science. 1996;31(18):4951-4955.

9. Damjanovic D, Brem F, Setter N. Crystal orientation dependence of the piezoelectric $\mathrm{d}_{33}$ coefficient in tetragonal $\mathrm{BaTiO}_{3}$ as a function of temperature. Applied Physics Letters. 2002;80(4):652-654.

10. Budimir M, Damjanovic D, Setter N. Piezoelectric anisotropyphase transition relations in perovskite single crystals. Journal of Applied Physics. 2003;94(10):6753-6761. 